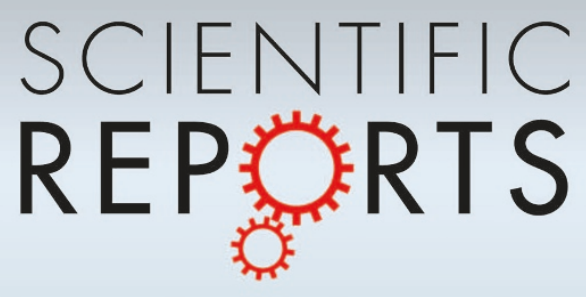

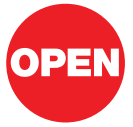

SUBJECT AREAS:

SOCIAL

ANTHROPOLOGY

BIOLOGICAL ANTHROPOLOGY

SOCIAL EVOLUTION

PSYCHOLOGY

Received

13 December 2012

Accepted

6 March 2013

Published

21 March 2013

Correspondence and requests for materials should be addressed to

M.E.B. (meb299@ cornell.edu)

\section{Humans use Compression Heuristics to Improve the Recall of Social Networks}

\author{
Matthew E. Brashears \\ Cornell University Department of Sociology Uris Hall Rm. 323 Ithaca, NY 14853.
}

The ability of primates, including humans, to maintain large social networks appears to depend on the ratio of the neocortex to the rest of the brain. However, observed human network size frequently exceeds predictions based on this ratio (e.g., "Dunbar's Number"), implying that human networks are too large to be cognitively managed. Here I show that humans adaptively use compression heuristics to allow larger amounts of social information to be stored in the same brain volume. I find that human adults can remember larger numbers of relationships in greater detail when a network exhibits triadic closure and kin labels than when it does not. These findings help to explain how humans manage large and complex social networks with finite cognitive resources and suggest that many of the unusual properties of human social networks are rooted in the strategies necessary to cope with cognitive limitations.

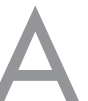

significant body of work has examined the connection between physical brain structure and social networks ${ }^{1-4}$, arguing that the size and structure of the human brain is related to Homo sapiens' social style of life ${ }^{5,6}$. This research program has identified a correlation between neocortical volume and social group size $e^{7-11}$, as well as differentiated "layers" of associates ${ }^{12}$. However, it has devoted less attention to the human use of mental frameworks, known as schemas ${ }^{13,14}$, to improve the efficiency of information processing and storage $^{15,16}$. Social information is processed and stored in a fundamentally different way than non-social informa$\operatorname{tion}^{17,18}$, but the utility of schemata extends to improving human learning speed for social information ${ }^{19,20}$. Here I show that schemata also function as compression heuristics, allowing individuals to discard information on the specific ties in a social network in favor of rules that permit the ties to be reconstructed from partial information. Schemata permit larger amounts of social information (the existence of relationships as well as their type) to be stored in the same brain mass and help explain why predictions of human network size based on brain characteristics (e.g., "Dunbar's Number") have often proven to be underestimates ${ }^{21-24}$. While the raw power of brain "hardware" is important, the efficiency of the schemata "software" is the key to the management of large and complex social networks.

Schemata are mental frameworks that organize the processing of information and influence its recall from memory ${ }^{13,16}$. Schemata that are useful for compressing social information can be strong (i.e., contain an extensive set of rules for reconstructing the network) or weak (i.e., contain fewer rules), and fall into two broad categories: "structural schemata" that are derivable from a network graph itself and "cultural schemata" that are provided by cultural expectations. Structural schemata are learned from regularities in a social network graph and a network is "structurally reducible" if it contains such features (e.g., reciprocity). The same structural schemata should appear in most, if not all, human societies because they stem from properties of the graph and are thus widely available for discovery. Cultural schemata are not derivable from the network structure and must be known to, and activated by, the individual a priori. These schemata indicate how individuals of particular types should be linked, and while cultural schemata vary from culture to culture (e.g., kinship systems vary by culture), the existence of schemata is universal (e.g., all cultures contain a kinship system). However, if the individual activates a cultural schema not appropriate to a given graph, particularly an incorrect strong cultural schema, the schema will degrade recall because the individual expects connections that may not exist.

This paper tests the effectiveness of two schemata that are operative in both modern and pre-modern societies $^{25}$ : triadic closure, a structural schema, and kin labels, a cultural schema. Beginning with the structural schema, consider a simple network consisting of three people: Alice, Bob, and Carol; if the network is characterized by triadic closure then we know that if Alice shares a tie with Bob (A-B), and Bob shares a tie with Carol (B-C), then Alice must share a tie with Carol (A-C). Thus, it is only necessary to remember two ties in a given triad as the third can be inferred, and if multiple triads are linked together the same dyad(s) may be implied by several different incomplete triads. 
Kinship labels (e.g., father/son) represent a strong cultural schema because they induce clear expectations about how actors should be tied (e.g., the brother of your husband is your brother-in-law) and imply additional information about network members that precludes certain relationships (e.g., a younger person cannot be the parent of an older person). In contrast, non-kin recreational labels (e.g., friend, club member) represent a weaker cultural schema, inducing fewer and less specific expectations about how actors are connected and conveying no additional information about actor traits.

I hypothesize that triadic closure will consistently improve recall of the target network. Because family relations are typically dense ${ }^{26}$ and kin labels are often inherently triadic (e.g., niece, mother-in-law, etc.), I also hypothesize that kin labels will improve recall when paired with triadic closure, and will degrade recall when not paired with triadic closure. Finally, the use of schemata to improve recall should produce characteristic errors when the structure of the network is not completely consistent with the schema. I therefore hypothesize that networks characterized by triadic closure or kin labels will be recalled with larger numbers of incorrectly closed triads.

\section{Results}

Experimental structure. A randomized laboratory experiment using two crossed manipulations to produce four conditions was employed to determine the extent to which triadic closure and kin labels improve the recall of social information. All four conditions presented a vignette describing a network of relationships between fifteen individuals (e.g., "Henry is Alyssa's brother. Henry is also Elizabeth's son..."), and each vignette included a structural reducibility manipulation and a cultural schema strength manipulation. A network was reducible if it contained triadic closure (Figure 1a), while it was irreducible if it contained no closed triads (Figure 1b). A network had a strong cultural schema if relations were described using kin labels (e.g., parent/child, brother/sister), and it had a weak cultural schema if the relations were described using non-kin recreational labels (e.g., friend, group member). Spouses were considered to be kin because they are fictive kin, because they often share genetic relationships to shared children, and because they represent a durable kin-like alliance.

All conditions contained 15 nodes, but all reducible conditions contained 46 reciprocated directed ties while all irreducible conditions contained 26 reciprocated directed ties and no condition contained unreciprocated ties. These constraints yielded network densities $^{27}$ of 0.219 and 0.124 , respectively. Because the number of nodes was constant and the specified constraints were imposed (e.g., triadic closure), the reducible and irreducible conditions were forced to have unequal numbers of ties, but the larger number of ties in the reducible conditions meant that there was more information to be recalled, thus penalizing the hypotheses.

The experiment included three parts: presentation of the randomly chosen vignette, completion of a standard measure of working memory capacity that also functioned as a clearance task, and an attempt to reproduce the network depicted in the vignette. Participants were informed that their compensation was contingent on their success in the recall phase but in fact all participants were compensated equally, with the deception ensuring that all participants were engaged by the task.

Measures. The primary outcome variable, performance, is the product of participant accuracy (the number of ties correctly recalled by a participant divided by the total number of ties recalled) and participant coverage (the number of ties correctly recalled by the participant divided by the total number of ties depicted in the vignette). This measure equals one when the participant recalls all the ties depicted in the vignette, and only those ties, and is less than one when the respondent recalls less than the full number of ties, or recalls ties that were not depicted. This measure compensates for the

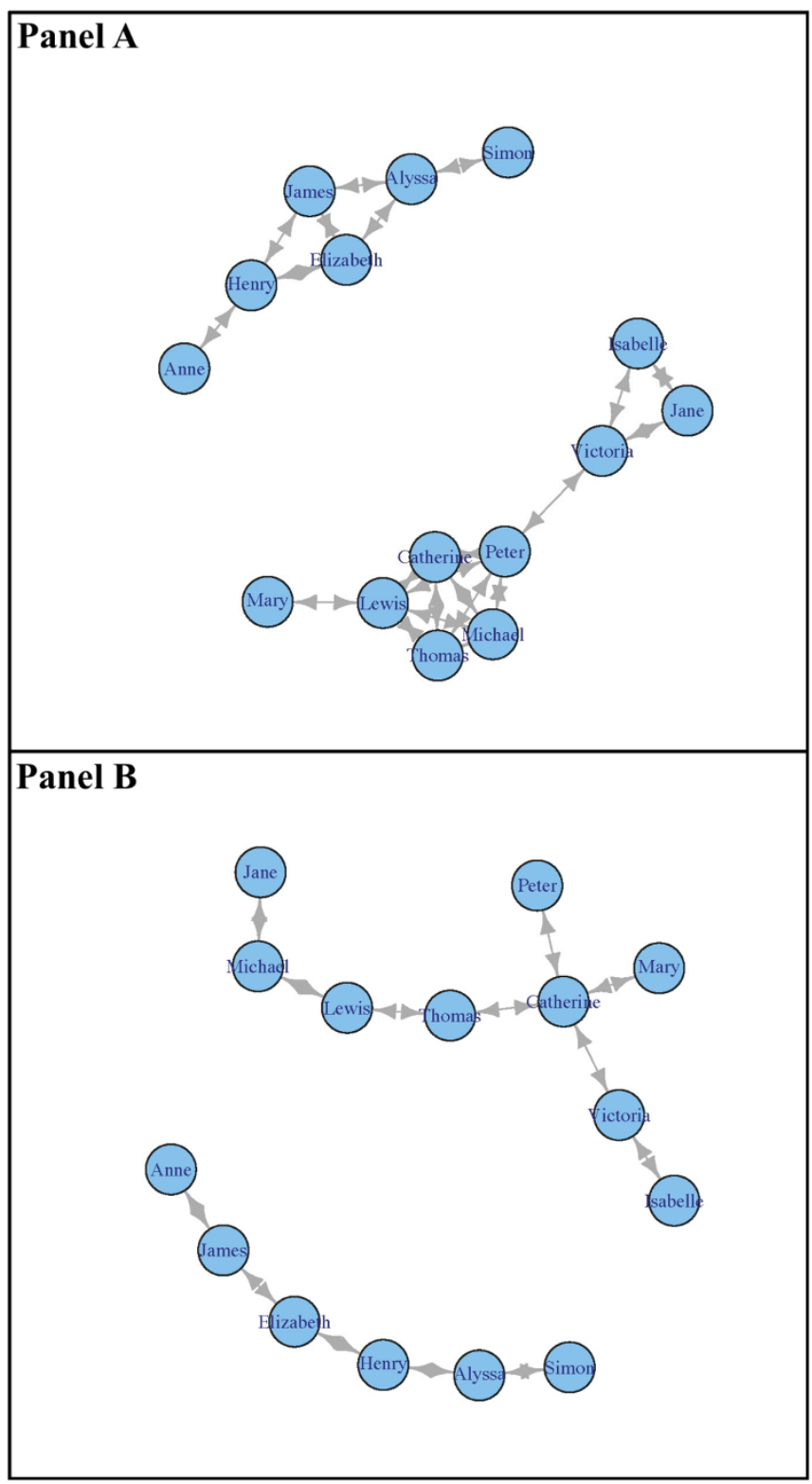

Figure $1 \mid$ Network structure depicted in the reducible (Panel A) and irreducible (Panel B) conditions.

unequal number of ties by condition and focuses on the presence of ties, which participants were instructed to recall, rather than on the absence of ties.

Participants also indicated the nature of each tie (e.g., spouse, friend) that they recalled. Because the correct answer is undefined for ties that were not given in the vignette, relationship accuracy is the number of correctly recalled ties that were assigned their correct relationship type, divided by the total number of correctly recalled ties.

Two additional measures, word span and time spent, were also included as controls. Word span is the participant's score on the working memory exercise and provides a conventional measure of general memory acuity, independent of the experimental task. Time spent is the number of seconds participants devoted to learning the vignettes.

Recall of ties: Performance. Table 1 provides relevant summary statistics. Because the mean amount of time participants spent studying the vignette is correlated with experimental condition, statistical tests are only performed using multivariate models. 
Performance varies from a high of 0.60 in the reducible/strong condition to a low of 0.34 in the irreducible/strong condition, matching predictions. Matching theoretical expectations, participants devoted the most time to studying the irreducible/strong vignette and the least time to studying the reducible/strong vignette, implying that they experienced these to be, respectively, the most difficult and least difficult conditions.

Table 2 (Model 1) regresses performance on the reducibility and schema strength manipulations, on an interaction between the manipulations, and on controls for word span and time spent. Performance is superior $(b=0.128 \pm 0.042, \mathrm{p}<0.01)$ in reducible networks and poorer $(b=-0.087 \pm 0.042, p<0.05)$ in strong schema networks. When the target networks are both reducible and strong schema, however, performance increases $(b=0.171 \pm$ $0.06, p<0.01$ ), and the magnitude of the interaction between these variables overwhelms the negative main effect of a strong schema. As predicted, reducibility aids recall overall, but a strong cultural schema is a detriment to recall when in the absence of reducibility and an asset to recall when in the presence of reducibility; when both are present recall performance increases by approximately fifty percent (i.e., twenty-one performance points).

Recall of ties: accuracy and coverage. The performance metric used above is the product of two separate measures: accuracy and coverage. While performance is the most reliable measure of recall success, accuracy and coverage are also analyzed separately below to provide additional detail. Accuracy is defined as the number of ties correctly recalled by a participant divided by the total number of ties recalled by the participant. A value of accuracy near one indicates that nearly all ties reported by the participant were present in the vignette, while a value near zero indicates that nearly all ties reported by the participant were not present in the vignette. Coverage is defined as the number of ties correctly recalled by the participant divided by the total number of ties depicted in the vignette. A value of coverage near one indicates that the participant successfully recalled nearly all of the ties depicted in the vignette, while a value near zero indicates that the participant recalled nearly none of the ties depicted in the vignette. An additional quantity, over guess, defined as the number of ties recalled by the participant, regardless of correctness, divided by the total number of ties present in the vignette, is included in all models. A value of over guess equal to one indicates that the participant recalled as many ties as were actually present; a value over one indicates recall of more ties than were present; and a value under one indicates recall of fewer ties than were present. Because eager reporting will tend to inflate coverage and deflate accuracy, the measures must be evaluated while controlling for over guess. Performance is not systematically influenced by eager reporting and thus is preferred as the main measure.

Accuracy (Table 1) varies from a high of 0.76 in the reducible/ weak condition to a low of 0.52 in the irreducible/strong condition, largely as predicted. Coverage varies from a high of 0.76 in the reducible/strong condition to a low of 0.52 in the irreducible/strong condition, precisely matching predictions.

Table 2 (Models $2 \& 3$ ) regresses accuracy and coverage on the reducibility and schema strength manipulations, on an interaction between the manipulations, as well as on controls for over guess, word span, and time spent. Participants are more accurate $(\mathrm{b}=0.144 \pm 0.04, \mathrm{p}<0.001)$, and have higher coverage $(\mathrm{b}=0.126 \pm 0.03, \mathrm{p}<0.001)$, in reducible networks and are less accurate $(b=-0.073 \pm 0.04, p<0.05)$, and have lower coverage $(b=-0.067 \pm 0.03, p<0.05$, one tailed $)$ in strong schema networks. When the target networks are both reducible and strong schema, however, the level of accuracy $(b=0.113 \pm 0.05, p<0.05)$ and coverage $(b=0.129 \pm 0.05, p<0.01)$ both increase, and the magnitude of the interaction between reducibility and schema strength is sufficient to overwhelm the negative main effect of a strong schema 


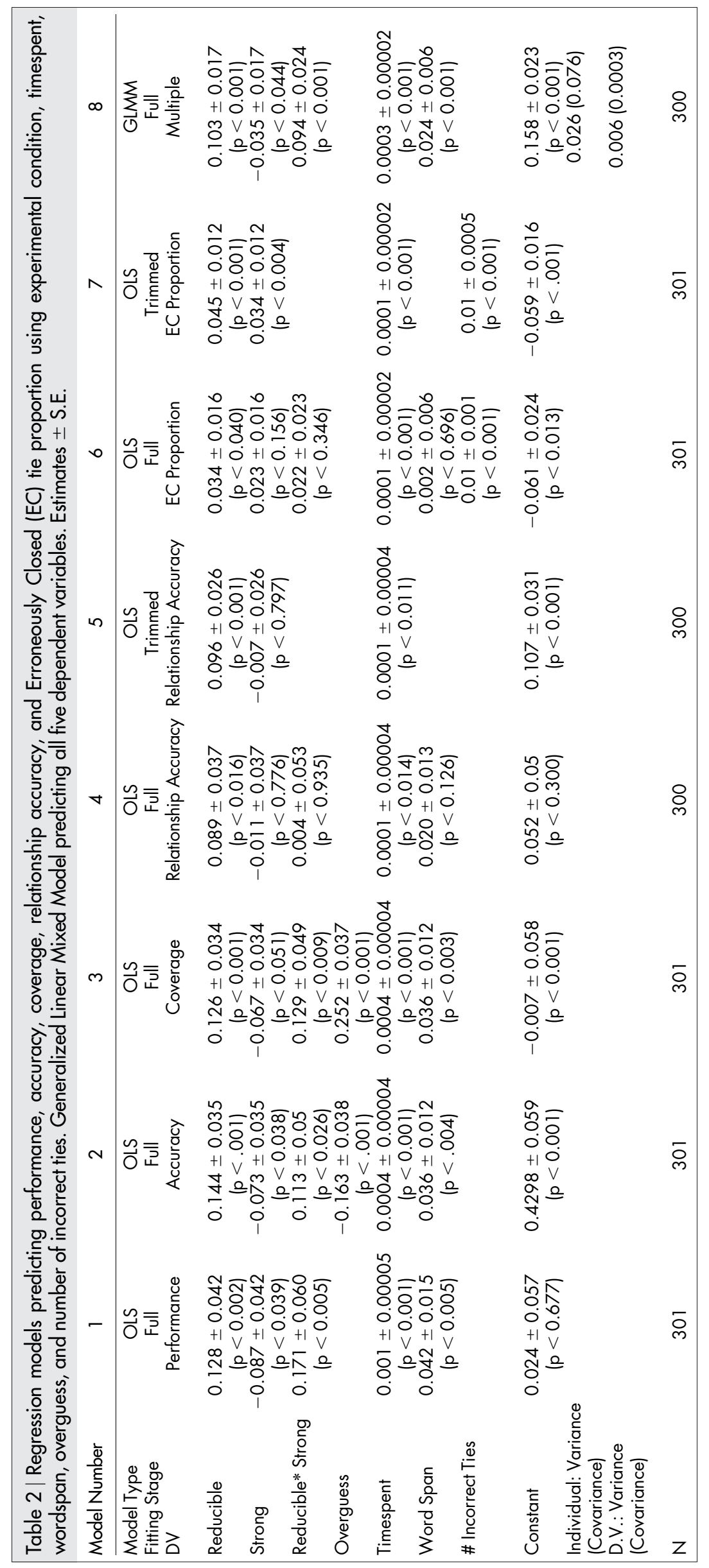


Table 3 | Summary statistics for errors ( \pm S.D.)

\begin{tabular}{lccccc} 
& Possible Incorrect Ties & Mean Incorrect Ties & Possible EC Ties & Mean Observed EC Ties & Mean EC Tie Proportion \\
\hline Reducible/Strong & 164 & $15.18 \pm 18.62$ & 32 & $6.57 \pm 8.33$ & $0.21 \pm 0.26$ \\
Reducible/Weak & 164 & $9.42 \pm 9.28$ & 32 & $3.41 \pm 3.36$ & $0.11 \pm 0.10$ \\
Irreducible/Strong & 184 & $13.07 \pm 11.10$ & 28 & $3.75 \pm 3.34$ & $0.13 \pm 0.12$ \\
Irreducible/Weak & 184 & $9.42 \pm 8.49$ & 28 & $2.04 \pm 2.29$ & $0.07 \pm 0.08$ \\
\hline
\end{tabular}

in both models. The results indicate that reducibility aids recall, but that a strong schema is a detriment to recall when in the absence of reducibility, and becomes an asset to recall when in the presence of reducibility, as hypothesized.

Recall of relationships: Accuracy. The best recall of relationship types (Table 1) is in the reducible/weak condition, while the worst recall is in the irreducible/strong condition, largely as predicted. Table 2 contains a regression of relationship accuracy on the reducibility and schema strength manipulations, an interaction between the manipulations, as well as the other variables. Any non-significant variables, both controls and interaction effects, are eliminated from the full model to produce a trimmed model (Table 2, Model 5), and the results indicate that recall of relationship types is better in the reducible conditions $(b=0.096 \pm 0.026, \mathrm{p}<0.001)$, but it is not consistently impacted by schema strength or the interaction of the manipulations. Consistent with expectations, while the reducible conditions contain more relations to be recalled, participants in these conditions are still able to correctly recall a larger proportion of the relationship types.

Error patterns. Thirty-two triads in the reducible conditions and twenty-eight triads in the irreducible conditions were left incomplete, but could be closed with the addition of a single tie. Because networks in the reducible condition contained closed triads, and because family structures tend towards triadic closure, the presence of reducibility or a strong cultural schema should increase the likelihood of recalling these "erroneously closed" (EC) ties. The presence of EC ties also helps distinguish the use of schemata to compress social information from an innate bias. If there is a general bias towards triadic closure, EC ties should be common in all conditions. However, if recall depends on the schema used, EC ties should be more common in the reducible and strong schema conditions than in the irreducible and weak schema conditions. The tendency to report EC ties was measured as the number of EC ties recalled by the participant divided by the number possible in their condition. Table 3 contains the relevant summary statistics. The largest mean proportion of EC ties is observed in the reducible/strong condition while the smallest proportion is observed in the irreducible/weak condition, as predicted.

The EC tie proportion was regressed on both the reducibility and schema strength manipulations, the interaction between the manipulations, the other variables introduced previously, and a new variable measuring the total number of erroneous ties of any type recalled by the participant, which controls for the propensity to make all kinds of mistakes. In the trimmed model (Table 2, Model 7), both reducible conditions $(b=0.045 \pm 0.012, p<0.001)$ and strong schema conditions $(b=0.034 \pm 0.012, \mathrm{p}<0.01)$ predict larger proportions of EC tie errors. When in conditions that include triadic closure, or when primed by a schema that implies triadic closure, participants are more likely to erroneously close triads. This pattern of errors indicates that the detected improvements in recall derive from the use of schemata, that participants recognize circumstances when particular schemata should be employed, and that expectations of triadic closure do not stem from an innate bias in human social reasoning.
Confirmatory modeling. Each of the preceding measures (i.e., performance, accuracy, coverage, relationship accuracy, and EC tie proportion) are calculated using the same participants and measure different aspects of recall success. As a result these measures are not mutually independent. I therefore reshape the data into long format and estimate a Generalized Linear Mixed Model (GLMM) that both permits each of the five dependent variables to co-vary and includes random effects at the individual level. This produces a single set of coefficients predicting all five dependent variables with the assumption that those variables are correlated. The results (Table 2, Model 8) confirm that reducibility has a generally positive effect $(b=0.103 \pm 0.017, p<0.001)$, a strong schema has a generally negative effect $(b=-0.035 \pm 0.017, \mathrm{p}<0.045)$, and that the interaction between them $(b=0.094 \pm 0.024$, $\mathrm{p}<0.001)$ is both positive and large enough to overwhelm the negative main effect of a strong schema, as expected. Thus, reducibility and schema strength have a consistent impact on the dependent variables even when their mutual dependence is taken into account. Moreover, as in the majority of the earlier models, reducibility improves recall success in all cases, while a strong schema only improves recall when in the presence of reducibility and degrades it otherwise. This model confirms that reducibility and schema strength have robust effects on the ability of humans to encode and recall social network information.

In Movie 1, ties are depicted when a specific percentage or more of respondents agree that they are present. This percentage, referred to as consensus, ranges from a high of $100 \%$ (i.e., all participants agree that a tie exists) to a low of $5 \%$ (i.e., $5 \%$ or more of the participants agree that a tie exists). The sooner a tie appears, the higher the degree of consensus that exists among participants about that relationship, and the easier it is to recall. Ties in the reducible conditions achieve substantially higher levels of consensus than do ties in the irreducible conditions. The first incorrect ties appear in the irreducible/strong condition at $30 \%$ consensus, both reducible conditions begin to exhibit EC ties at $20 \%$ consensus, and incorrect ties linking the components appear in all graphs at $10 \%$ consensus and below.

\section{Discussion}

These findings show that humans adaptively make use of schemata as compression heuristics to store social information more efficiently. This permits humans to develop and maintain social networks that are substantially larger than might be expected based on their neocortical ratios, and further shows that the absolute processing capacity of the brain is often not as important as its efficiency.

Networks that can be more easily represented in the mind (e.g., adhere to schemata) can reach larger absolute sizes and provide a competitive advantage ${ }^{28}$. As a result there may have been selective pressure on human groups favoring certain types of network structures over others. Humans may therefore have evolved constraints on structure in order to acquire larger networks, and many of the unique features of human social networks ${ }^{29}$ may derive from the methods used to reduce the associated cognitive burden.

Triadic closure and kin labels can be used to compress social information, but many other schemata may have similar benefits. Structural balance ${ }^{30}$, for example, is a tendency for individuals to like those whom their friends like, and dislike those whom their friends 
dislike. A preference for balance is difficult to explain, however, because liking someone whom your friends dislike allows one to reap rewards by acting as a go-between ${ }^{31}$. However, structural balance is advantageous cognitively because the views of your associates match yours, reducing the number of relations to be tracked from $\mathrm{n}(\mathrm{n}-1)$ to only $\mathrm{n}-1$. We might thus expect balanced relations to be preferred because they simplify the cognitive demands of maintaining relationships.

Schemas can be used to improve the storage and recall of network information, thereby permitting humans to maintain larger and more complex social networks. The capability to identify and employ such schemas is thus adaptive, regardless of the specific biological relevance of the schemas themselves. By analogy, the ability to acquire behaviors can be adaptive even if some of the behaviors that are acquired are not immediately relevant to survival or reproduction. Likewise, the ability to use schemas reduces the cognitive burden on the individual even if the schemas themselves are not biologically relevant. Some schemas are likely to be biologically relevant (e.g., kin relations capture genetic relatedness and mating alliances) and, as a result, are probably common to many human populations. Nevertheless, care should be exercised with this logic as even kinship systems, which are based to some extent on genetic relatedness, vary culturally. Investigation of biologically relevant schemas would thus be valuable to understanding how humans represent and maintain networks, but must be sensitive to cultural flexibility.

Because in this study the recall phase was separated from the presentation phase by a clearance task, schemata must be exerting their effect by improving the recall of information from long-term storage. However, additional research will be necessary to determine whether these improvements stem from changes to how ties are encoded into long-term storage or changes to how information is accessed from long-term storage. If schemata influence how information is encoded, the cognitive burdens on the executive function are likely experienced when the network is initially learned. In contrast, if schemata are used to reconstruct the network when longterm storage is accessed, the recall phase is likely to be the most cognitively demanding.

The use of schemata improves the recall of social information, but individuals likely differ in their skill at encoding networks using schemas. Persons with greater skill may be able to maintain larger or more complex networks than those with less skill, thereby acquiring and maintaining advantageous, but cognitively burdensome, network positions. Such advantages would not need to be deliberately sought, as persons with greater ability would simply equilibrate at more complex networks. Similarly, individuals with various social deficits (e.g., social anxiety or autism spectrum disorder) may be less able to identify and apply schemata than cognitively typical others. This deficit would force individuals to learn more ties by rote and to expend more effort on processing social information in working memory, thereby raising their level of stress. Because processing occurs in real time, individuals with relatively less ability to use schemas would likely equilibrate at more peripheral network positions where the complexity is low enough to be managed.

Finally, other species exhibit cultural behavior ${ }^{32}$, attempt to model the intentions of others ${ }^{33}$, and use graph features to draw social inferences ${ }^{34}$. It may therefore be possible to adapt this methodology to study other organisms, allowing researchers to determine if the use of schemata for compressing social information is uniquely human or something shared with other species. At the same time, existing research suggests that much of the improvement in processing efficiency has likely occurred in Homo. For example, based on previous research ${ }^{7}$ the +2.26 increase in neocortical ratio from Avahi to Pan (i.e., across the range of extant non-human primates) should permit Pan to manage 64 more direct ties and roughly 4,200 more indirect ties. By comparison, the +0.88 increase in neocortical ratio from Pan to Homo sapiens should permit Homo sapiens to manage 83 more direct ties and approximately 17,600 more indirect ties. While it is not necessarily the case that individuals are actively tracking every potential indirect relation, it is nevertheless the case that the smaller increase in neocortical ratio from Pan to Homo produced a larger gain in social capability than did the larger increase from Avahi to Pan. The ability to use schemata to more efficiently store social information may be the adaptation that has permitted modern humans to improve their cognitive efficiency by such a considerable margin. In addition, fossil evidence ${ }^{12}$ suggests sharp and significant increases in group size with the appearance of Homo ergaster and Homo heidelbergensis, implying that the necessary adaptations may be primarily or solely represented in Homo. In any investigation of non-humans it will also be necessary to take group structure into account, as this will substantially alter the complexity of the problem and by extension the adaptive benefit of schemata. Organisms that are in constant contact with only minimal need to coordinate (e.g., flocking birds) are less likely to need such cognitive improvements than organisms with semi-independent groups that exhibit complex coordination (e.g., fission-fusion structures).

\section{Methods}

Participants (197 female, 104 male) were recruited from among the undergraduate population of Cornell University using flyers and other direct solicitations and were randomized into one of four conditions with a minimum of 72 and a maximum of 78 participants per condition. Participants began the presentation phase by sitting at a prepared computer terminal and answering a series of simple demographic questions. A randomly chosen vignette was then presented as a paragraph of text and the participants were instructed to commit it to memory. All participants were told that the amount of compensation they would receive for completing the study was contingent on their success at recalling the vignette, but in fact all participants were compensated equally. The deception ensured that the participants were motivated to recall the information accurately. Vignettes were used because (1) they permit absolute control of the depicted network, (2) they allow the dyads to be presented in context, and (3) humans routinely exchange social information linguistically (e.g., gossip). The network size of 15 was chosen using research on working memory capacity ${ }^{35}$ with the intention of stressing the participants; the number of individuals depicted exceeds the estimated maximum capacity of working memory by roughly a factor of two, and the potential number of relations (i.e., 210) is more than an order of magnitude greater. All vignettes contained two disconnected components (i.e., subgroups with no connections between them), and the strong and weak schema conditions used different relationship types (e.g., spouse, friend, etc.). The same number of relationship types were used in each vignette, the components did not vary in size by condition, and both characters and ties were presented in the same order in each vignette. The schema strength manipulation only impacted the terms used to describe the network and did not impact its structure. One vignette was presented to each participant and all participants who were in the same condition saw the same vignette. Participants had unlimited time to study the vignette and were allowed to take notes, but knew that the notes would be confiscated before the recall phase. The amount of time spent studying the vignette was measured without the participants' knowledge.

Once the participant finished studying the vignette, the middle phase began and participants completed a word span exercise ${ }^{36}$ with the experimenter. The participant read a series of sentences out loud and, at pre-determined times, recalled the last word in each preceding sentence. The exercise continued until the participant was unable to recall the words or obtained the maximum score. The sentences were drawn from popular press books and all contained between 13 and 16 words.

In the recall phase participants checked a series of boxes to indicate which characters had relations with each other. Participants then indicated the type of relationship (e.g., spouse, friend) they believed characterized each tie they identified in the preceding set of questions. Participants could return to the first part of the recall phase and change their selections as often as desired, but this cleared the relationship type choices, and participants received no feedback on their answers. Finally, participants were compensated and debriefed. The experiment typically required forty minutes to complete, and all participants completed it. All procedures were approved by the Cornell IRB and all subjects gave their informed consent.

1. Bickart, K. C., Wright, C. I., Dauthoff, R. J., Dickerson, B. C. \& Barrett, L. F. Amygdala volume and social network size in humans. Nat. Neurosci. 14, 163-164 (2010)

2. Meyer, M. L., Spunt, R. P., Berkman, E. T., Taylor, S. E. \& Lieberman, M. D. Evidence for social working memory from a parametric functional MRI study. Proc. Natl. Acad. Sci. USA 109, 1883-1888 (2012).

3. Sallet, K. et al. Social network size affects neural circuits in macaques. Science 334, 697-700 (2011)

4. Zahn, R. et al. Social concepts are represented in the superior anterior temporal cortex. Proc. Natl. Acad. Sci. USA 104, 6430-6435 (2007). 
5. Humphrey, N. K. The social function of intellect. Growing Points in Ethology, ed. P. P. G. Bateson \& R. A. Hinde, pgs. 303-317. Cambridge: Cambridge University Press (1976).

6. Silk, J. B. Social Components of Fitness in Primate Groups. Science 317, 1347-1351 (2007)

7. Dunbar, R. I. M. Neocortex size as a constraint on group size in primates. Journal of Human Evolution 20, 469-493 (1992).

8. Dunbar, R. I. M. Coevolution of neocortical size, group size and language in humans. Behavioral and Brain Sciences 16, 681-735 (1993).

9. Dunbar, R. I. M. Neocortex size and group size in primates: a test of the hypothesis. Journal of Human Evolution 28, 287-296 (1995).

10. Goncalves, B., Perra, N. \& Vespignani, A. Modeling Users' Activity on Twitter Networks: Validation of Dunbar's Number. PLOSone 6, doi:10.1371/ journal.pone.0022656 (2011).

11. Stiller, J. \& Dunbar, R. I. M. Perspective-taking and memory capacity predict network size. Social Networks 29, 93-104 (2007).

12. Dunbar, R. I. M. Bridging the bonding gap: the transition from primates to humans. Phil. Trans. Roy. Soc. B, 367, 1837-1846 (2012).

13. Bartlett, F. C. Remembering: A Study in Experimental and Social Psychology. Cambridge, MA: Cambridge University Press (1932).

14. Neisser, U. Cognitive Psychology. New York: Appleton-Crofts (1967).

15. Martin, C. L. New directions for investigating children's gender knowledge. Developmental Review 13, 184-204 (1993).

16. Brewer, W. F. \& Treyens, J. C. Role of Schemata in Memory for Places. Cognitive Psychology 13, 207-230 (1981).

17. Janicik, G. A. \& Larrick, R. P. Social Network Schemas and the Learning of Incomplete Networks. Journal of Personality and Social Psychology 88, 348-364 (2005).

18. Simpson, B., Markovsky, B. \& Steketee, M. Power and the perception of social networks. Social Networks 33, 166-171 (2011).

19. De Soto, C. B. Learning a Social Structure. Journal of Abnormal and Social Psychology 60, 417-421 (1960).

20. Picek, J. S., Sherman, S. J. \& Shiffrin, R. M. Cognitive Organization and Coding of Social Structures. Journal of Personality and Social Psychology 31, 758-768 (1975).

21. Bernard, H. R., Shelley, G. A. \& Killworth, P. How Much of a Network does the GSS and RSW Dredge Up? Social Networks 9, 49-63 (1987).

22. McCarty, C., Killworth, P. D., Bernard, H. R., Johnsen, E. \& Shelley, G. Comparing Two Methods for Estimating Network Size. Human Organization 60, 28-39 (2001).

23. DiPrete, T. A., Gelman, A., McCormick, T., Teitler, J. \& Zheng, T. Segregation and Social Networks Based on Acquaintanceship and Trust. American Journal of Sociology 116, 1234-1283 (2011)

24. Killworth, P. D., Johnsen, E. G., Bernard, H. R., Shelley, G. A. \& McCarty, C. Estimating the size of personal networks. Social Networks 12, 289-312 (1990).
25. Apicella, C. L., Marlowe, F. W., Fowler, J. H. \& Christakis, N. A. Social networks and cooperation in hunter-gatherers. Nature. 481, 497-501 (2012).

26. Marsden, P. V. Core Discussion Networks of Americans. American Sociological Review 52, 122-131 (1987).

27. Wasserman, S. \& Faust, K. Social Network Analysis: Methods and Applications. Cambridge: Cambridge University Press. Pgs. 101-103 (1999).

28. DeScioli, P. \& Kurzban, R. The Alliance Hypothesis for Human Friendship. PLoS ONE 4, doi:10.1371/journal.pone.0005802 (2009).

29. Newman, M. E. J. \& Park, J. Why social networks are different from other types of networks. Phys. Rev. E 68, Doi: 10.1103/PhysRevE.68.036122 (2003).

30. Heider, F. The Psychology of Interpersonal Relations. New York: Wiley (1958).

31. Burt, R. S. Structural Holes: The Social Structure of Competition. Cambridge: Harvard University Press (1992).

32. Mann, J., Stanton, M. A., Patterson, E. M., Bienenstock, E. J. \& Singh, L. O. Social networks reveal cultural behaviour in tool-using dolphins. Nat. Comms. 3 , doi:10.1039/ncomms1983 (2012).

33. Myowa-Yamakoshi, M., Scola, C. \& Hirata, S. Humans and chimpanzees attend differently to goal-directed actions. Nat. Comms. 3, doi:10.1038/ncomms1695 (2012).

34. Paz-y-Mino, C, G., Bond, A. B., Kamil, A. C. \& Balda, R. P. Pinyon jays use transitive inference to predict social dominance. Nature 430, 778-781 (2004)

35. Reisberg, D. Cognition: Exploring the Science of the Mind. New York: W. W. Norton \& Company (1997).

36. Daneman, M. \& Carpenter, P. A. Individual Differences in Working Memory and Reading. Journal of Verbal Learning and Verbal Behavior 19, 450-466 (1980).

\section{Acknowledgements}

Thanks to Emily Hoagland, Laura Aufderheide Brashears, Elisa Bienenstock, Haim Bar, Jon Kleinberg, Brent Simpson, Edward Lawler, Brian Rubineau, Ben Cornwell, Eric Gladstone, Tiffany Ramsay, Neil Lewis, Joy Jiang, Soojin Park, Tom Seo, Khadija Ahmed, Matthew Sloan, Bethany Nichols, Shannon Frank and Jordan Raddick for their contributions. This research was supported by the USA National Science Foundation (Award \#1059282).

\section{Additional information}

Competing financial interests: The author declares no competing financial interests.

License: This work is licensed under a Creative Commons

Attribution-NonCommercial-NoDerivs 3.0 Unported License. To view a copy of this license, visit http://creativecommons.org/licenses/by-nc-nd/3.0/

How to cite this article: Brashears, M.E. Humans use Compression Heuristics to Improve the Recall of Social Networks. Sci. Rep. 3, 1513; DOI:10.1038/srep01513 (2013). 